\title{
THOTH: Um Assistente Cognitivo com Small Talks para Conversação Tutorial
}

\begin{abstract}
This paper presents a brief description of THOTH (Training by Highly Ontology-oriented Tutoring Host), a cognitive assistant for tutorial conversation for learning and teaching processes, capable to enunciate small talks for better interactivity with students $A$ theoretical basis, including tool structure and interface are introduced, as well as preliminary results from a perception survey that allow one to demonstrate the potential of THOTH's usage.
\end{abstract}

Resumo. Este artigo apresenta uma breve descrição de THOTH (Training by Highly Ontology-oriented Tutoring Host), um assistente cognitivo para conversação tutorial em processos de ensino e aprendizagem, capaz de enunciar small talks para melhorar a interatividade com o estudante. São mostradas bases teóricas, a estrutura da ferramenta e da interface e alguns resultados preliminares de uma pesquisa de percepção que permitem demonstrar o potencial da ferramenta.

\section{Introdução}

$\mathrm{O}$ desenvolvimento de sistemas robustos de Q\&A (question and answer, pergunta $\mathrm{e}$ resposta), tal como o IBM Watson (High, 2012), tem grande potencial para uso na educação. Q\&A consiste em uma forma especial de recuperação da informação para construção de conhecimento unificando linguagem natural com a representação de conhecimento, inferência lógica e busca semântica (Bhattacharyya, 2013). THOTH (acrônimo para Training by Highly Ontology-oriented Tutoring Host) foi modelado como uma ferramenta para o aprendizado autônomo por parte do aluno de conteúdos organizados em cursos ou disciplinas. O modelo de conhecimento usa ontologias de domínio, construídas na forma de triplas objeto-atributo-valor (OAV). As perguntas formuladas em linguagem natural são analisadas conforme quatro métricas de distância de palavras, que infere sobre o objeto indagado e o atributo respectivo. A busca é efetuada nas ontologias, recuperando-se as respostas que se referem ao conteúdo solicitado, utilizando um tipo de diálogo baseado, portanto, em perguntas e respostas, com as perguntas default sugeridas por THOTH e as respostas geradas de forma estocástica, conforme os valores das triplas OAV presentes nas ontologias.

Small talks foi objeto de estudo primeiramente na área de Linguística e se referem a pequenas conversações cujo propósito é manter um canal de comunicação aberto entre os interlocutores, suavizando a interatividade por intermédio do uso de falas e tópicos que são comuns na conversação humana. São utilizadas, por exemplo, para abrir ou fechar uma conversação, lançar tópicos não relacionados diretamente ao contexto principal da fala e também para evitar pausas constrangedoras. Bickmore e Cassell (1999) propuseram uma interface de conversação de small talks e narrativas para facilitar a interação com os usuários. Portanto, técnicas baseadas no uso de small talks poderiam facilitar a interatividade e auxiliar no aprendizado pela oferta de um contexto emocional positivo para ferramentas de ensino e aprendizagem. 
VI Congresso Brasileiro de Informática na Educação (CBIE 2017)

Anais do XXVIII Simpósio Brasileiro de Informática na Educação (SBIE 2017)

\section{Bases Teóricas}

THOTH é concebido como um assistente cognitivo ou de aprendizado, com o objetivo de fornecer conhecimento de um domínio específico. Seu uso é justificado como uma ferramenta de apoio com dois objetivos básicos, devidamente fundamentados na taxonomia de objetivos de aprendizagem de Bloom (Bhattacharyya, 2013): i) remembering, onde se incluem os comportamentos que dão ênfase ao recordar unidades de informação suscetíveis de assimilação; e ii) understanding, onde há expectativa do entendimento dos conteúdos transmitidos, para a posterior utilização das ideias e conceitos aprendidos.

A efetividade das interações de um usuário com uma ferramenta da classe dos chatterbots está relacionada à possibilidade do usuário perceber uma "mente" do outro lado da interface, em conformidade com a teoria da postura intencional de Daniel Dennett (Dennett, 2006). A estratégia básica da postura intencional é tratar a entidade que é parte da conversação como um agente capaz de predizer e então explicar suas ações e movimentos.

O uso de small talks em THOTH compreende 12 segmentos baseados na categorização de Schneider (1988): abertura, encerramento, autoconhecimento, quebra de silêncio, tempo das consultas, frases retóricas, sugestões de conteúdo, gerenciamento da aprendizagem, retomada, confirmação de objetos e demonstrações de gentileza.

\section{Estrutura da Interface de Usuário}

THOTH é operacionalizado por meio de arquitetura cliente-servidor, sendo instalado em um servidor Java/Linux disponibilizado em intranet e permitindo conexão de múltiplos usuários em browser. A interface (Figura 1) é dividida em 5 blocos, incluindo o bloco de conversação em modo chatterbot. Os blocos de gestão do aprendizado, questões e mensagens de interação possuem itens predefinidos para acesso rápido por parte do aluno. Os objetos são apresentados de forma hierárquica para serem escolhidos pelo aluno. Existe também um bloco de complemento, onde são apresentadas imagens, vídeos, fotos ou animações que complementam a conversação efetuada por THOTH.

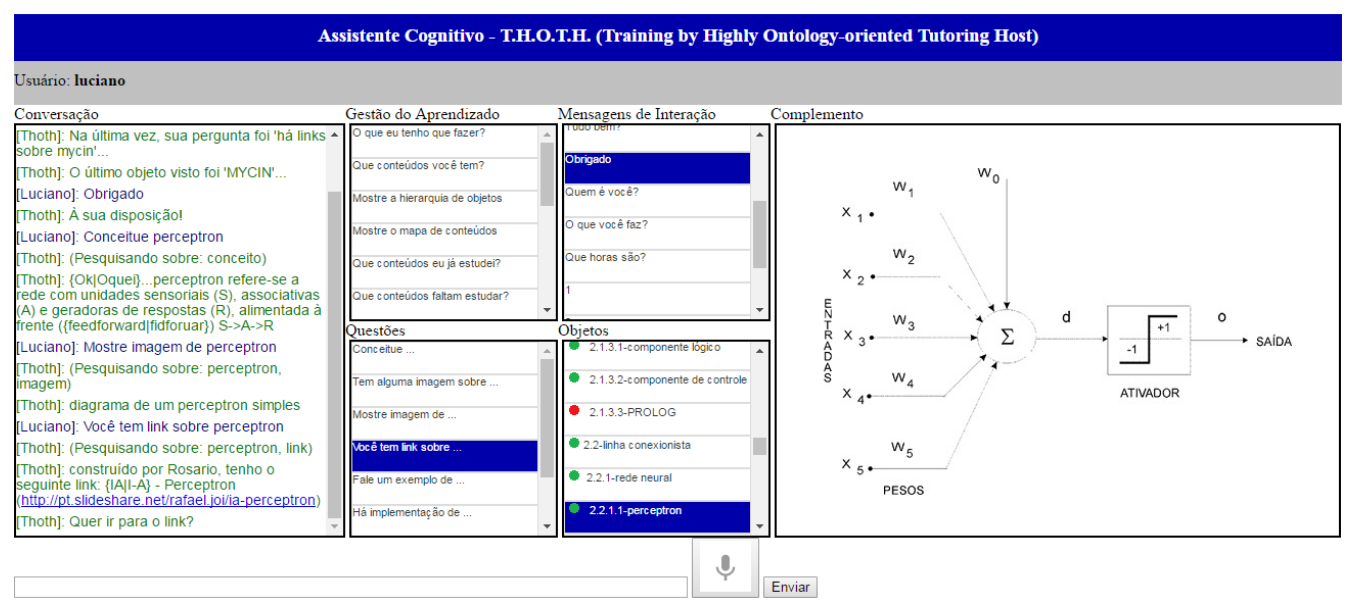

Figura 1: Interface de THOTH com um exemplo de interação.

$\mathrm{O}$ aluno pode solicitar a THOTH os exercícios para praticar sua aprendizagem, os quais são relacionados aos objetos existentes nas ontologias, permitindo uma avaliação de 
VI Congresso Brasileiro de Informática na Educação (CBIE 2017)

Anais do XXVIII Simpósio Brasileiro de Informática na Educação (SBIE 2017)

aprendizagem por objeto. THOTH utiliza esta informação e repassa como feedback ao aluno, indicando quais objetos ele precisa concentrar mais sua atenção.

\section{Resultados Parciais}

Seguindo uma metodologia de avaliação de percepção da ferramenta, THOTH foi introduzido para uma turma da disciplina de Inteligência Artificial Aplicada de um curso presencial de graduação com 19 estudantes. Uma explicação sobre a ferramenta foi feita preliminarmente, contemplando propósito, funcionalidades e exemplos de conversação. Na sequência, os estudantes interagiram com a ferramenta pelo período de uma hora e meia, sendo mostrado a seguir um questionário com 12 questões com escala Likert valoradas em escala crescente de percepção de 1 a 5, mais uma questão aberta.

Para a análise dos dados, foram criadas duas categorias conforme as informações fornecidas pelos alunos. A categoria "interação" estava relacionada com características tais como usabilidade e possibilidades de comunicação, bem como referências para melhoria da interface. Algumas características foram bem avaliadas, como o grau de compreensão das respostas dadas por THOTH $(4,62)$, e o potencial demonstrado para explicar conceitos $(4,25)$. Entretanto, a visão de THOTH como uma ferramenta de suporte teve um escore mais baixo $(3,42)$. A categoria "intencionalidade" se embasava na postura intencional de Dennett para a identificação de aspectos humanos na interlocução. Dentro desta categoria, foram bem avaliados a percepção de "inteligência" de THOTH $(3,83)$ e a sensação de falar com uma pessoa $(3,75)$. Entretanto, a sensação de manifestação de uma personalidade ficou com um escore mais baixo $(2,75)$.

\section{Considerações Finais}

A pesquisa efetuada com os estudantes indicou um bom potencial para uso, ainda que certos elementos devam ser aprimorados na ferramenta. Em trabalhos posteriores serão analisadas as conversações em nível de detalhe maior, de maneira a permitir o delineamento de um perfil do uso de small talks.

\section{Referências}

Bhattacharyya, P. (2013). A Survey on Question Answering System. Retrieved from http://www.cfilt.iitb.ac.in/resources/surveys/Question Answering Surveybiplab.pdf

Bickmore, T., \& Cassell, J. (1999). Small Talk and Conversational Storytelling in Embodied Conversational Interface Agents. Proceedings of the AAAI Fall Symposium on Narrative Intelligence, 87-92.

Dennett, D. C. (2006). Brainstorms: escritos filosóficos sobre a mente e a psicologia. São Paulo: UNESP.

High, R. (2012). The Era of Cognitive Systems: An Inside Look at IBM Watson and How it Works. International Business Machines Corporation, 1(1), 1-14. Retrieved from http://www.redbooks.ibm.com/redpapers/pdfs/redp4955.pdf

Schneider, K. P. (1988). Small talk: Analyzing phatic discourse. Marburg, Germany: Hitzeroth. 